\title{
THE INDIANA UNIVERSITY MIDWEST PROTON RADIATION INSTITUTE
}

\author{
V. Anferov, M. Ball, G.P. Berg, B. Broderick, J. Collins, G. East, D. Friesel, D. Jenner, W.P. Jones, \\ J. Katuin, S. Klein, C. Nelson, N. Schreuder, Wm. Starks, J. Self \\ IUCF, Bloomington, IN 47408, USA
}

\section{Abstract}

Funding to convert the IUCF K220 separated sector cyclotrons into a dedicated proton source for Cancer Therapy was released by Indiana University in August of 2000. Phase I of the Midwest Proton Radiation Institute (MPRI) will initially consist of the IUCF separated sector cyclotrons upgraded to operate at a fixed energy of 205 $\mathrm{MeV}$, a treatment room containing both a general purpose large field horizontal fixed line and a small field line for the treatment of eye melanoma, a second treatment room with a $360^{\circ}$ rotating Gantry, and a comprehensive medical clinic. A third treatment room with a gantry is incorporated into the beam delivery system design as a future upgrade. The MPRI beam delivery system is now under construction and will incorporate a beam sharing system to allow simultaneous beam delivery to all medical treatment rooms, as well as to medical and commercial research facilities. Additional Funding is anticipated for the construction of a dedicated Radiation Effects Research (RERP) facility for NASA and other commercial users requiring beam similar to those used for proton therapy.
This report will outline the goals and design of the MPRI medical and commercial facilities, its present construction status and projected completion schedules.

\section{FACILITY DESCRIPTION}

The Indiana University Cyclotron Facility (IUCF), shown in Fig. 1, houses two separate accelerator systems for research and applications. The first is a synchrotron based nuclear physics research facility made up of an intense ( 1 $\mathrm{mA}$ Peak) polarized proton and deuteron ion source (CIPIOS) [1], a $2.4 \mathrm{Tm}$ booster synchrotron (CIS) [2] and a 3.6 Tm electron cooled synchrotron storage ring (Cooler) [3]. The second system consists of a $600 \mathrm{keV}$ Cockroft-Walton pre-accelerator, a K15 separated sector injector cyclotron and a K220 separated sector main cyclotron [4]. When the NSF funded research program for the cyclotrons ended in 1998, IUCF was already using the cyclotron facilities for a variety of commercial, biomedical and radiation research applications. A strong RERP program had been operating since 1995 [5], and a

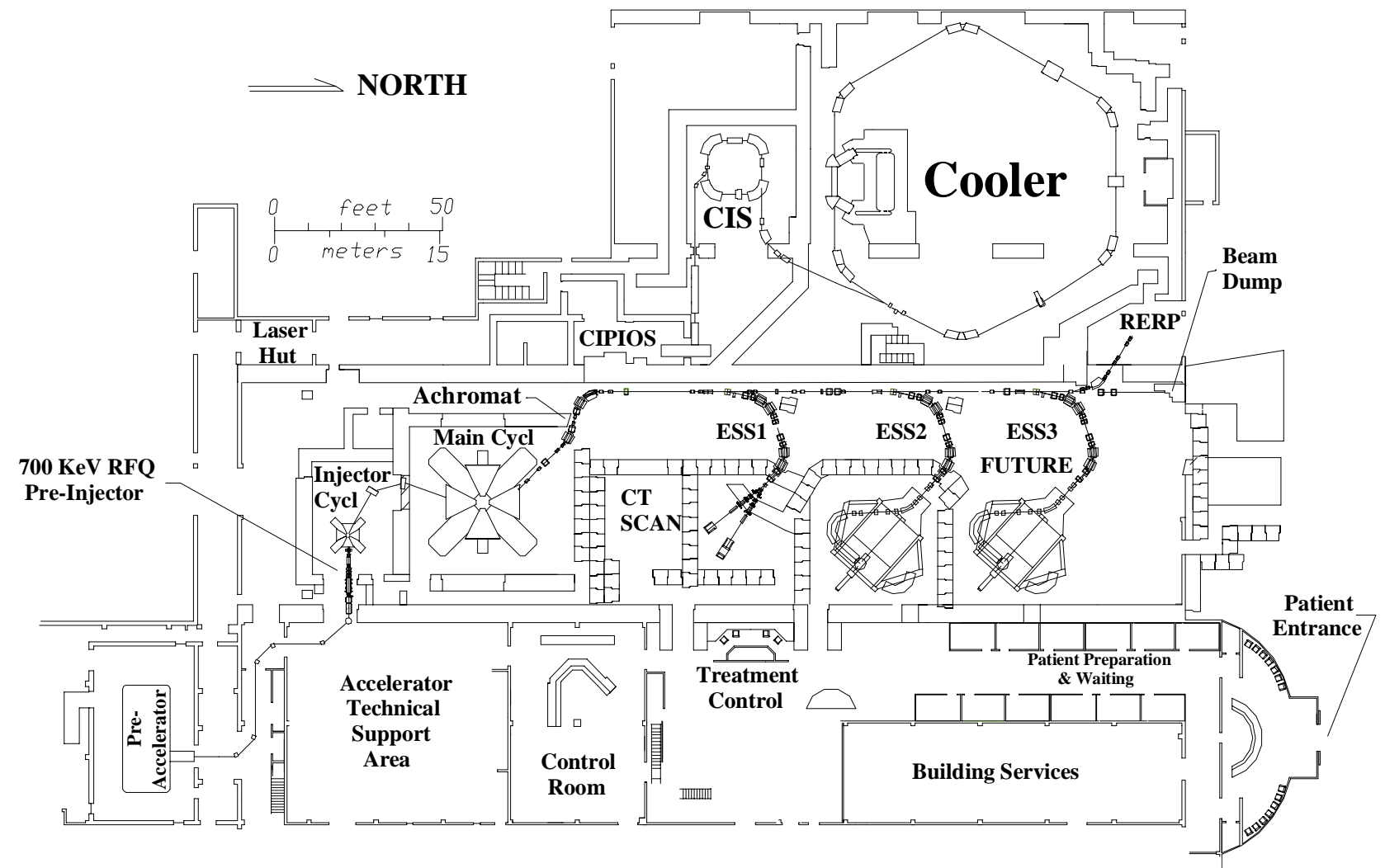

Figure 1. The IUCF Facilities showing the Synchrotrons, Cyclotrons, and MPRI medical facilities. 
pilot horizontal eye line for the treatment of choroidal neovascular membrane (CNVM) associated with Age Related Macular Degeneration (AMD) was performing clinical trails [6]. The fixed horizontal eye line and nuclear research facilities were dismantled in FY 2000, and temporary facilities were installed to continue commercial use of the cyclotrons for radiation effects and biomedical research activities during the conversion to the medical treatment facilities illustrated in Fig. 1.

\section{UPGRADES AND OPERATIONS}

A historical review of the reliability and performance limitations of the IUCF cyclotrons revealed several areas where improvements could significantly enhance their effectiveness as a proton source for Cancer Therapy. Operating experience at IUCF and elsewhere has shown that frequent energy changes, like those needed for routine operations for nuclear research, precipitate both beam stability and accelerator hardware reliability problems. Consequently, the IUCF cyclotrons will be operated continuously at a fixed energy of $205 \mathrm{MeV}$ for MPRI. Beam energy variability will be accomplished with an energy degrader at the entrance of the beam transport lines to each of the three planned treatment rooms to permit rapid setup and delivery of proton beams between 60 and $205 \mathrm{MeV}$ to each treatment room.

Two major hardware sources of downtime during IUCF's peak operating years (over 7000 hrs annually from 1983 to 1993) were the atomic beam polarized ion source, and the $600 \mathrm{kV}$ Cockroft-Walton pre-accelerator systems, which together accounted for half the $10 \%$ of scheduled operations lost to breakdowns. Polarized protons are not required for Cancer Therapy, and a more reliable proton volume source is now used. The $600 \mathrm{kV}$ ion source terminal experienced voltage holding and regulation problems, and will be replaced in early 2002 with a commercial $750 \mathrm{keV}$ Radio Frequency Quadrupole (RFQ) pre-injector. The stability of an RFQ coupled with the increased injection energy will significantly improve the reliability and reproducibility of cyclotron beam operations. The design for the new $750 \mathrm{keV}$ RFQ and low energy beam transport system was provided by AccSys Technology, Inc [7] and were previously reported [8].

A new cyclotron control computer and Vsystem software package were installed and commissioned in March 2001. New controls hardware (DAC/ADC/PLC) will be installed in late 2001, and are similar in design to the systems developed for the 2.4 Tm CIS booster synchrotron commissioned in 1998 and more recently installed on the "Cooler" in 2000 [9]. These new cyclotron controls are the first part of a more global MPRI treatment control system.

Other cyclotron system upgrades include re-alignment of the main cyclotron extraction system, re-tuning both cyclotron rf amplifiers for peak operation at $35.52 \mathrm{MHz}$ (205 MeV protons), replacement of all cyclotron and beam line de-ionized water cooling systems, and the addition of new beam diagnostic devices (BPM's, wire HARPS and scanners, phase probes) to aid in the set-up and monitoring of beams for proton therapy. These and other smaller upgrades are designed to provide the $95 \%$ beam delivery reliability specified for MPRI operations.

\section{MPRI BEAM DELIVERY SYSTEMS}

Construction of the MPRI beam delivery system began with the installation of a $14.3 \mathrm{~m}$ long achromatic beam line at the exit of the main cyclotron (see fig. 1). This will feed a $57 \mathrm{~m}$ long "Trunk Line" which ends at a beam dump at the north end of the cyclotron hall. The doubly achromatic waist at the end of the Achromat is reproduced near the entrance to each of the three energy selection beam lines (ESS1, 2, \& 3). Since the cyclotron will be operated at a fixed energy of $205 \mathrm{MeV}$, the achromat and trunk beam lines are constructed using non-laminated quadrupole and dipole magnets recovered from the original cyclotron beam lines dismantled last year. The trunk line beam dump will house a multi-layer Faraday cup [10] to continuously monitor the cyclotron beam energy with a resolution of about $100 \mathrm{keV}$.

Three beam splitting systems, each consisting of a fast ferrite kicker and Lambertson dipole magnet [11], are used to switch $205 \mathrm{MeV}$ protons from the trunk into each of the ESS lines on demand. An energy degrader is located at an achromatic double waist immediately following the Lambertson dipole in each ESS line to vary the proton beam energy between 205 to $60 \mathrm{MeV}$. Consequently, the ESS lines are constructed using laminated dipole and quadrupole elements to facilitate rapid energy changes during clinical operations. The ESS lines again deliver a doubly achromatic beam to the entrance of each treatment room. The beam splitting, energy degrading and ESS transport systems permit the simultaneous delivery of pulsed beam to each treatment room with its own energy, intensity and distribution. This is essentially equivalent to having a cyclotron for each treatment room.

Beam diagnostics in the MPRI beam delivery systems include non-destructive beam position and intensity monitors (BPM's), multi-wire Harps and wire scanners for beam intensity and position measurement, and multi-leaf Faraday cups and time-of-flight (TOF) devices for energy measurement. Feedback hardware and software will be used to automatically keep the beam steered through the center of the trunk line to the beam dump. A TOF monitor in the BL3 Achromat and the multi-layer Faraday cup in the trunk line beam dump and ESS line will be used to set up and maintain a constant energy beam in the ESS lines during treatment. All beam splitting, diagnostic, and feedback systems have been developed and used at IUCF during the previous 25 years of operations for nuclear research.

The Achromat line was commissioned in Nov, 2000. Installation of the trunk line, beam dump and ESS1 beam line to the fixed horizontal beam line treatment room is in progress and completion is anticipated before the end of 2001. Phase I construction plans call for the completion of the ESS1 line and the fixed horizontal beam treatment room and clinical facilities in 2003, and the ESS2 beam line and Gantry room in late 2004. 


\section{TREATMENT ROOM FACILITIES}

The first MPRI treatment facility to become operational at IUCF will be a fixed horizontal beam line treatment room. It will initially be fitted with both large and small field nozzle designs, as shown in fig. 1, based on two treatment facilities previously constructed and used at IUCF. A large field general purpose line for head and neck cancers [12], and a small field low energy proton therapy nozzle previously used for AMD clinical trials conducted at IUCF between 1998 and 2000 [6] will be installed and accessed via a $\pm 10^{\circ}$ switch magnet at the entrance of this treatment room. Beam wobbling will be used in the large field line, and wobbler magnet designs are now being considered.

The second treatment room to become operational will contain a $360^{\circ}$ rotating gantry, also shown in Fig. 1. Two 40'x29'x12' deep concrete lined pits are being dug into the floor of the IUCF cyclotron experimental hall to house the gantry support structures. These are large enough to house most known commercially available gantry designs. Formal requests for price quotations for a commercial gantry structure and beam transport system are being processed, with the intent to purchase a gantry by early fall of 2001. A 30 ton bridge crane is available in the cyclotron hall, and covers the location of the gantry pit. IUCF and MPRI may elect to fabricate the gantry beam transport system and nozzle in-house.

A commercial Motoman UP200 robot is being adapted to precisely position patients in both MPRI treatment rooms. Commercial robots are relatively inexpensive, are engineered with excellent precision, and have an extensively documented operational history. The MPRI patient positioning system will utilize three independent technologies to assure patient safety and/or position accuracy. The robot will translate the patient from a docking station to a predetermined location in the beam coordinate system. An optical tracking system will follow the robot movement, provide a collision safety mechanism, and direct the robot to compensate for differences between the predicted patient position and actual position by using patient specific markers. Digital radiography will be used to verify the accurate location of the biological target at the beam iso-center, and direct any fine adjustments required to match the treatment prescription. In addition, the robot will be fitted with accelerometers and inclinometers to further verify the internal robot guidance system readout and assure the safety of the patient.

\section{MEDICAL FACILITY DEVELOPMENT}

Medical facilities will be constructed to provide support for the clinical application of the cyclotrons. The clinic will be housed largely within the existing structure, making use of the areas previously occupied by nuclear physics experimental stations and the "low bay" research support area. The layout is based upon a wheel and hub configuration that allows the staff to be centrally located. The floor in the clinic area will be raised approximately two feet above the existing concrete floor to allow conduit, ductwork, plumbing and cable accessibility, ease of access to the beam line in the treatment rooms, and increased cushioning to reduce job related fatigue. Patients will enter MPRI by way of a new parking lot, lobby and reception area to be constructed at the north end of the building. IUCF staff will continue to use the parking facilities and entrance on the east side, upper level, facilitating the ease of operations of all IUCF supported activities. Construction of the clinical facilities will begin in mid 2002.

\section{RESEARCH APPLICATIONS}

An important aspect of IUCF's contribution to Proton Therapy is to increase biomedical and radiation effects understanding through innovative research and technical development. To enable the cyclotron facility to actively pursue applied research in radiation effects, research stations will be constructed at the north end of the medical facility. Variable energy proton beams will be routinely available at this station in a manner analogous to the treatment room delivery. Beam sharing will allow preclinical, radiation biology and applied research to continue as a priority interest of IUCF at all times when the cyclotrons are operating. Some examples of ongoing research efforts are: dose delivery verification, space related radiation effects and Single Event Upset research, and biomedical research activities.

\section{REFERENCES}

[1] V. Derenchuk et al, AIP Conf. 421, 422 (1998).

[2] D.L. Friesel et al, PAC'99, IEEE 99CH36366, 498 (1999).

[3] R.E. Pollock, PAC'89, IEEE 89CH2699-0, 17 (1989).

[4] R.E. Pollock, PAC'77, IEEE Trans. Nucl. Sci, Vol. NS-24, No. 3, 1505 (1977).

[5] C.C Foster et al, AIP Conf. 392, (1996) pp. 1131.

[6] C. Block et al, IUCF Publication, (1998).

[7] AccSys Technology, Inc, Pleasanton, CA. RHamm@Linacs.com

[8] D.L. Friesel et al, Proc. CAARI 2000, Denton Texas, Nov. 2000, to be published.

[9] J. Callahan et al, PAC'97, IEEE 97CH36167, 2449 (1997)

[10] A.N. Schreuder et al, PTCOG XXXII, Uppsala, SE, (2000) p 8.

[11] D.L. Friesel et al, $12^{\text {th }}$ Intl. Cyclotron Conf, Berlin (1989) pp 380-384.

[12] C. Block et al, NIM B79 (1993) pp890-894. 